\title{
Investigation of viscous fluid flow in an eccentrically deposited annulus using CFD methods
}

\author{
M. Bojko ${ }^{1, a}$, M. Kozdera ${ }^{1}$, M.Kozubkova ${ }^{1}$ \\ ${ }^{1, \mathrm{a}}$ Department of Hydromechanics and Hydraulic Equipment, Faculty of Mechanical Engineering, VŠB-Technical \\ University of Ostrava, 17. listopadu 15, Ostrava, Czech Republic
}

\begin{abstract}
The theory of fluid flow in an eccentrically deposited annulus has of great importance especially in the design of sliding bearings (axial, radial). If the geometry is more complex or shaft is deposited eccentrically, then a suitable alternative for design hydrostatic bearing is using ANSYS Fluent, which solves the general three-dimensional viscous fluid flow also in complex geometry. The problem of flow solves in the narrow gap between the cylinders in this paper, when the inner cylinder is stored with a defined eccentricity. The movement of the inner cylinder is composed of two motions (rotation, precession), i.e. rotation around its own axis and move along the circle whose radius is the size of the eccentricity. Addition the pressure gradient is considered in the axial direction. In the introductory section describes the methodology for defining of motions (rotation and precession of the inner cylinder) when the user function (UDF) is created that defines the rotation and move along the circle in $\mathrm{C}++$. The above described methodology of the solution was then applied to the $3 \mathrm{D}$ model with a defined pressure drop when the problem was solved as a time-dependent with a time value corresponding to two turns of the internal shaft.
\end{abstract}

\section{Introduction}

Sliding bearings surface represent two planar or cylindrical surfaces which are each other moving and engineering designs are based on the theory of flow in the gap between these surfaces [6]. If the geometry is complicated or there is eccentric shaft, then a suitable variant of hydrostatic bearings design the spatial viscous fluid flow solutions applicable in complex geometry using ANSYS Fluent is utilized. Thus, it can be very effectively to apply the design for different types of hydrostatic bearings (axial, radial, linear bearing, hydrostatic matrix) using the aforementioned program. The results can be used in the design of the seal in many applications, e.g. for pumps.

The challenge is to define a mathematical model of single-phase flow in a narrow gap between the cylinders with rotating of the inner one. This cylinder also held precession (circular motion). The inner cylinder is placed in the basic position with defined eccentricity. In the direction of the axis the pressure gradient is considered. The mathematical model of the flow is given by the equation of continuity and Navier - Stokes equations. The model is then solved numerically by finite volume method in ANSYS Fluent13.0 [4]. To create a geometry DesignModeler program was used and to create a computational grid ANSYS meshing.
Rotation and precession of the inner cylinder is given by user defined function (UDF) in $\mathrm{C}++$. Subsequently thus generated function is implemented in the program environment ANSYS Fluent. The user function is specified equations of inner cylinder center motion through equation of a circle, as it is also related to the subsequent definition of the moving computational grid (moving mesh). The above described solution methodology was tuned on a 2D model and then applied to the $3 \mathrm{D}$ model. In $3 \mathrm{D}$ model the role of the pressure gradient was also considered and the problem was solved as time-dependent. Time value corresponded to the two inner shaft speed. Numerical solutions were used to evaluate the size effect of rotation and precession in the radial and tangential forces acting on the shaft. The angular velocity of rotation and precession was entered in the range from $600 \mathrm{rev} / \mathrm{min}$ to $3000 \mathrm{rev} / \mathrm{min}$. For individual variations of numerical simulations the radial and tangential force components acting on the shaft, depending on the rotation were evaluated. Consequently, at the conclusion from the forces process the mean values are plotted in the graph, depending on the angular velocity of precession rotation at a constant size. The results show polynomial dependence of the radial force and the linear dependence of tangential forces on the rotation at precession constant.

a marian.bojko@vsb.cz 


\section{Geometry of eccentric narrow gap}

Solved geometry is presented on figure 1 and figure 2 . Region consists of outer and inner cylinder, which is placed eccentrically and input and output pocket is added. The main dimensions are shown on figure 2. Input of flowing medium (water) in the narrow gap is illustrated in figure 2 (blue) at a defined pressure. The inner cylinder rotates around its own axis and also held precession (moving along the circle with a given eccentricity). Detailed analysis of the movement is described in Chapter 4.

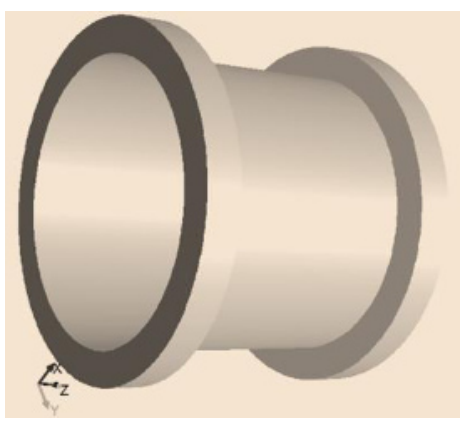

Fig. 1. Geometry of the computational domain

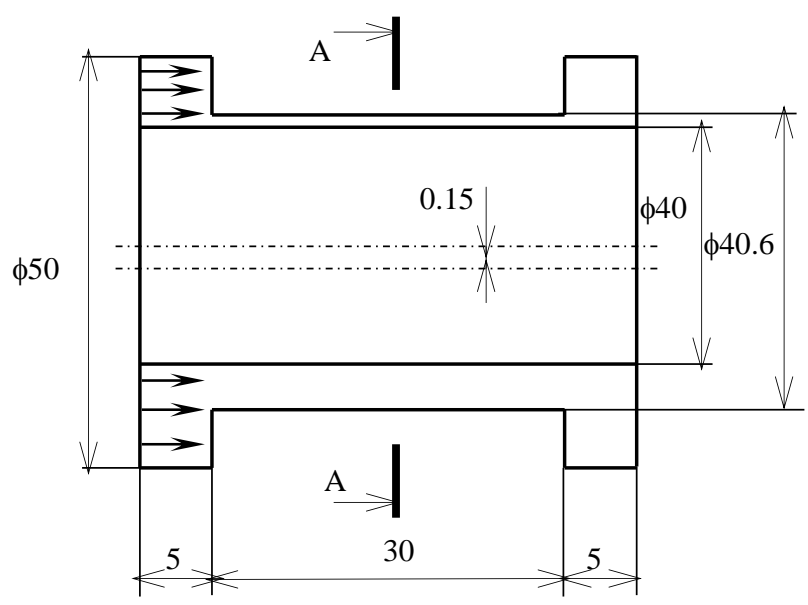

Fig. 2. Longitudinal section of the computational domain

\section{Mathematical model of flow in the narrow gap}

Due to the nature of the geometry the general threedimensional space is assumed. Type of flow is determined from the geometry and operating parameters and subsequently identified dimensionless numbers such as Reynolds number, the size of which determines the type of flow (laminar vs. turbulent) and Taylor number, which determines whether Taylor vortices arise in this gap between the cylinders at zero pressure gradient. The pressure gradient suppresses the existence of these vortices. The definition of these parameters in the case of flow between the cylinders with the rotation is:

Reynolds number:

$$
R e=\frac{\Omega r_{I}\left(r_{2}-r_{1}\right)}{v}
$$

where $(\Omega)$ is the angular velocity, $\left(r_{1}\right)$ is the inner radius $\left(r_{2}\right)$ is the outer radius, $(v)$ is the kinematic viscosity. The critical value of the transition from laminar to turbulent flow is in the interval 1100 to 1400 .

Taylor number :

$$
T a=\operatorname{Re} \sqrt{\frac{r_{2}-r}{r_{l}}}=\frac{\Omega r_{1} s}{v} \sqrt{\frac{r_{2}-r_{1}}{r_{l}}}
$$

where $(s)$ is $s=r_{2}-r_{1}$. Critical value characterizing the formation of stationary vortices is 41.6 and critical value of periodic wave modes of vortices is up to one hundred times of the critical Taylor number, see figure 3 .
A)

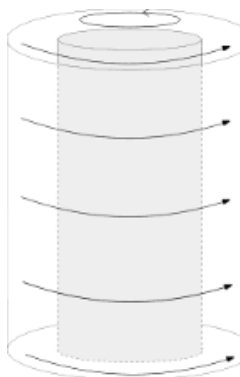

B)

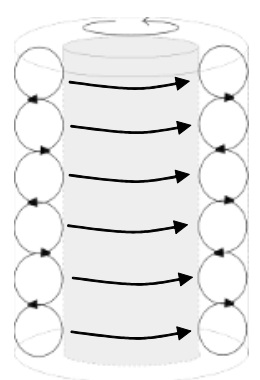

C)

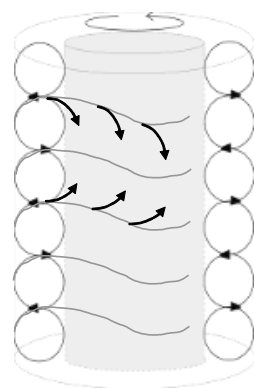

A) non-vortex flow, fluid flows in the tangential direction

B) stationary vortices

C) periodical non-stationary vortices

Fig. 3. Characteristics of flow between concentric cylinders with the inner cylinder rotation

In Table 1 there are tabulated values of the Reynolds and Taylor numbers for given speed and geometry, and the existence of vortices is defined here for the zero pressure gradient $(\Delta p=0 \mathrm{~Pa})$ and without eccentricity.

Table 1. The values of the Reynolds and Taylor numbers for given revolutions and geometry $r_{l}=0.0197 \mathrm{~m}, s=0.0003 \mathrm{~m}$ a pro $(\triangle p=O P a)$

\begin{tabular}{|c|c|c|c|c|}
\hline$[\boldsymbol{\Omega}]=\mathbf{r a d} / \mathbf{s}$ & $\boldsymbol{R e}$ & $\begin{array}{c}\text { Regime of } \\
\text { flow }\end{array}$ & $\boldsymbol{T a}$ & $\begin{array}{c}\text { Taylor } \\
\text { vortices }\end{array}$ \\
\hline 62.832 & 376.99 & laminar & 46.2 & - \\
\hline 125.664 & 753.98 & laminar & 92.3 & stationary \\
\hline 188.496 & 1130.97 & laminar & 138.5 & stationary \\
\hline 251.327 & 1507.96 & transient & 184.7 & stationary \\
\hline 303.687 & 1822.12 & transient & 223.2 & periodical \\
\hline 314.159 & 1884.96 & transient & 230.9 & periodical \\
\hline
\end{tabular}

The flow is supposed therefore in the region of laminar and transition region between laminar and turbulent flow. Based on experience with modeling of Taylor vortices it is usefulto use in those cases rather laminar flow mathematical model, since turbulence models too deformed the vortices due to artificial flow viscosity [5]. In test solution of 2D tasks turbulent mathematical model the turbulent viscosity was evaluated. That have been for water in the range from 0.01 to $0.025 \mathrm{~Pa} \cdot \mathrm{s}$, therefore 
very low. This means that the viscosity at molecular level again indicates the transition area. Due to the character of task the problem was solved as time-dependent.

Thus, the mathematical model of the flow is given by the equation of continuity and Navier - Stokes equations, when laminar flow is considered. The mathematical model is then solved in ANSYS Fluent13.0 [4].

Equation of continuity:

$$
\frac{\partial\left(u_{j}\right)}{\partial x_{j}}=0
$$

Navier-Stokes equation:

$$
\begin{aligned}
& \frac{\partial\left(u_{i}\right)}{\partial t}+\frac{\partial\left(u_{i} u_{j}\right)}{\partial x_{j}}=-\frac{1}{\rho} \frac{\partial p}{\partial x_{i}}+\frac{\partial}{\partial x_{j}}\left(\mu \frac{\partial u_{i}}{\partial x_{j}}\right)+ \\
& +\delta_{i 3} g+f_{c} \varepsilon_{i j 3} u_{j}+f_{i}
\end{aligned}
$$

\section{Creation of computational grid}

The problem is complicated in that it is expected to move the boundaries area that the outer cylinder does not rotate, but the inner rotating angular speed $\omega$, and moreover, moves in a circle with a given eccentricity (held precession angular velocity $\Omega$ ). Thus, it is necessary to consider a change of grid in each iteration step. Therefore, selected from various alternatives from slipping grid where each cylinder was surrounded by a layer of cells moving with them and between these layers were created annulus, which deforms and suitably also defines the interface between the layers of cells, see figure 4. Movement of the inner cylinder (rotation and precession in a circle) is defined by custom function in $\mathrm{C}$ ++ (UDF function). Number of cells along the height of the individual layers is at least the 10 .

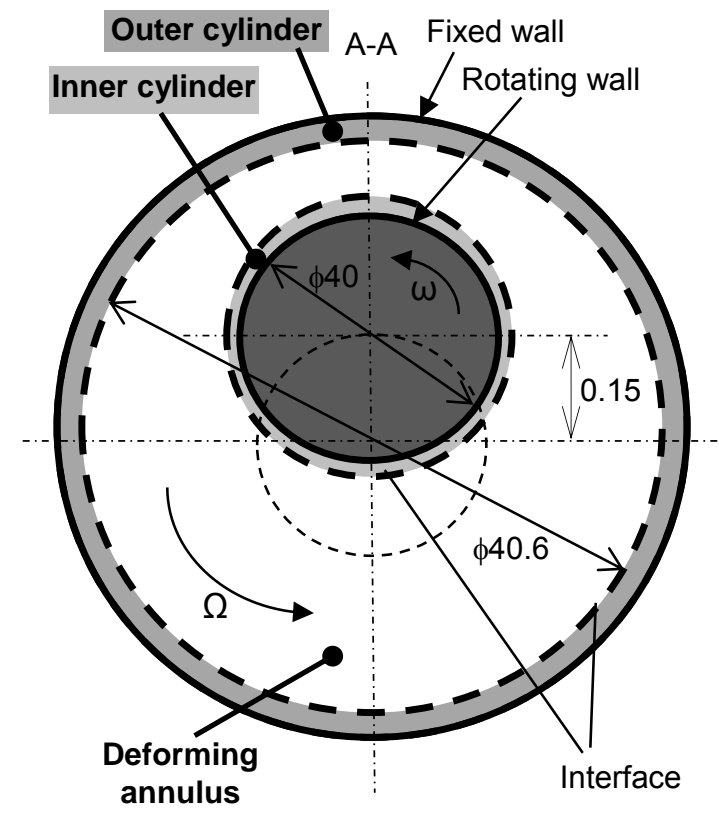

Fig. 4. Description of the problem of rotation, the computational domain

Then it can be determine that the total cell number was 1292500. User functions of rotation and precession was tested on a simple 2D area with respect to debugging functionality. Computing grid of individual areas 2D model are shown in figure 5. Subsequently debugged user function was used in the 3D model of narrow gap.

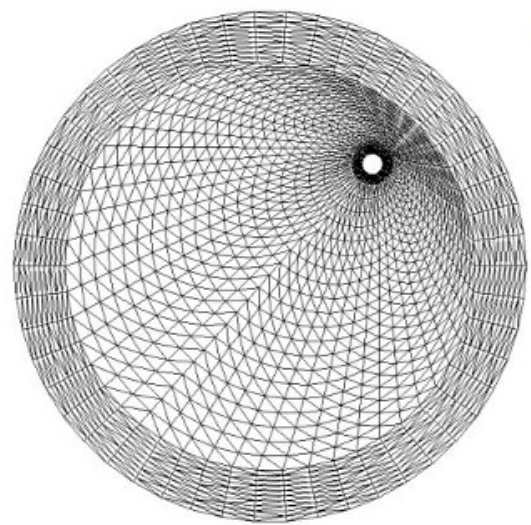

Fig. 5. Schematic representation of the computational grid on a simple 2D model

The resulting 3D model of computational grid of narrow gap including input and output is shown in figure 6. For the creation of computational grid was used software ANSYS Meshing. Computational grid consists of hexahedron with an adequate number of cells along the height of individual regions (min. 5 series). Dislocation cells along the height in each area are shown in figure 7 , figure 8. Details show the distribution of elements along the height of the outer areas. The inner cylinder is in the basic position is shifted by eccentricity $(0.15)$ which corresponds to the scheme (figure 3). Subsequently pictures figure 7, figure 8 illustrate a grid in this position (figure 7 - shows the upper area, and figure 8 lower area).

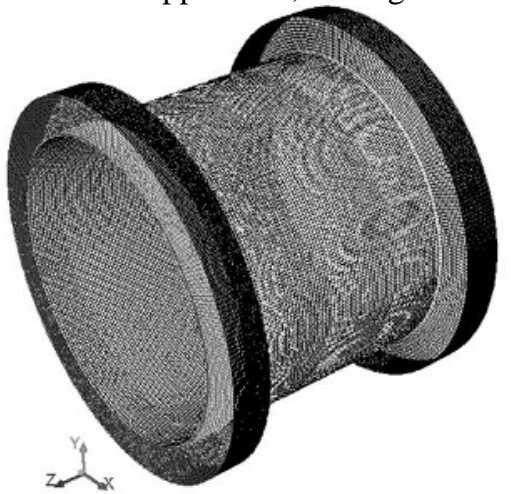

Fig. 6. Computational grid of 3D model narrow gap

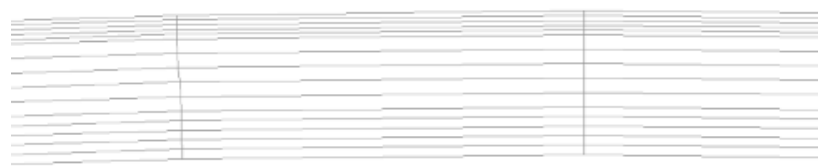

Fig. 7. Detail of computational grid in narrow gap (upper area)

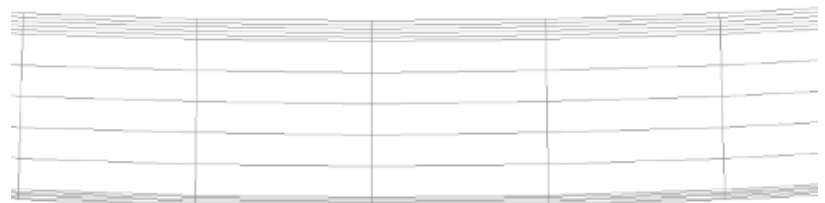

Fig. 8. Detail of computational grid in narrow gap (lower area) 


\section{Variants of numerical simulation, boundary conditions, physical properties}

Two variants of geometry have been defined for numerical simulation in ANSYS Fluent. The first option was a 2D model without considering the pressure drop (geometry corresponds to figure 4) and a second variant of the 3D model which is described in Chapter 2 (figure 1 , figure 2) with a defined pressure drop.

\subsection{Numerical solutions in 2D geometry without considering the pressure drop}

Variant 2D computational domain was used mainly to debug functionality mutual rotation and precession of the rotor. Computational domain is schematically illustrated in figure 5.

Boundary conditions for numerical solution:

- $\quad$ rotation of rotor $(\omega=62.8319 \mathrm{rad} / \mathrm{s}, 125.6637$ $\mathrm{rad} / \mathrm{s}, 188.4956 \mathrm{rad} / \mathrm{s}, 251.3274 \mathrm{rad} / \mathrm{s}, 314.1593$ $\mathrm{rad} / \mathrm{s}$ )

- $\quad$ precession of rotor $(\Omega=62.8319 \mathrm{rad} / \mathrm{s}, 125.6637$ $\mathrm{rad} / \mathrm{s}, 188.4956 \mathrm{rad} / \mathrm{s}, 251.3274 \mathrm{rad} / \mathrm{s}, 314.1593$ $\mathrm{rad} / \mathrm{s})$

In total, 25 variants defined of solutions (all combinations of rotation $\omega$ and precession $\Omega$ of rotor). The tasks were solved as a time-dependent with a defined time step. The total computing time corresponded to two speed of rotor about the axis of the computational domain (two precession movements). The aim of numerical solution was to evaluate the distribution of radial force $F_{r}$ and tangential force $F_{t}$ on the rotor, depending on the time or turn the rotor. In the software ANSYS Fluent13.0 can evaluate the force components $F_{x}$ and $F_{y}$. Subsequently, based on the conversion relationships can be determined forces $F_{r}, F_{t}$ acting on the rotor.

Radial force :

$F_{r}=F_{x} \cos ((\varphi+\pi / 2) t)+F_{y} \sin ((\varphi+\pi / 2) t)$

Tangential force:

$$
F_{t}=-F_{x} \sin ((\varphi+\pi / 2) t)+F_{y} \cos ((\varphi+\pi / 2) t)
$$

Note. $\pi / 2$ expresses the initial position of the rotor.

Size of the forces $F_{x}, F_{y}, F_{r}$ and $F_{t}$ were evaluated with the frequency of rotation of the rotor $\pi / 4$ for two turns. Subsequently from distribution of the radial and tangential forces depending on the rotation were determined mean values of $F_{r} F_{t}$ [1], [2], [3]. Thus defined procedure of evaluation forces was carried out for all combinations of rotation and precession.

\subsection{Numerical solution of the 3D geometry with consideration of pressure drop}

Debugging methodology for solving of flow in the 2D narrow gap was subsequently applied to the 3D model.
Evaluation of the radial and tangential forces was carried out in the same manner as described in the previous chapter. In terms of boundary conditions are also considered pressure drop along its longwise.

Boundary conditions for numerical solution:

- $\quad$ rotation of rotor $(\omega=62.8319 \mathrm{rad} / \mathrm{s}, 125.6637$ $\mathrm{rad} / \mathrm{s}, 188.4956 \mathrm{rad} / \mathrm{s}, 251.3274 \mathrm{rad} / \mathrm{s}, 314.1593$ $\mathrm{rad} / \mathrm{s}$ )

- $\quad$ precession of rotor $(\Omega=62.8319 \mathrm{rad} / \mathrm{s}, 125.6637$ $\mathrm{rad} / \mathrm{s}, 188.4956 \mathrm{rad} / \mathrm{s}, 251.3274 \mathrm{rad} / \mathrm{s}, 314.1593$ $\mathrm{rad} / \mathrm{s}$ )

- $\quad$ pressure inlet (pressure condition, $p_{\text {rel }}=10^{5} \mathrm{~Pa}$ )

- $\quad$ pressure outlet (pressure condition, $p_{\text {rel }}=0 \mathrm{~Pa}$ ) operation pressure $\left(p_{o}=101325 \mathrm{~Pa}\right)$

Flowing medium is water, which is defined by a constant value of the density $\rho=1000 \mathrm{~kg} \cdot \mathrm{m}^{-3}$ and a kinematic viscosity $v=10^{-6} \mathrm{~m}^{2} \cdot \mathrm{s}^{-1}$, thus defined incompressible flow.

\section{Evaluation of numerical simulation of 2D and 3D model of the flow in the narrow gap}

\subsection{Evaluation of forces $F_{x}, F_{y}, F_{r}$ a $F_{t}$ for 3D model of flow}

For each variant set of boundary conditions (rotation and precession rotor) was evaluated distribution of $F_{x}, F_{y}, F_{r}$ and $F_{t}$ depending on the rotor position for two turns. From the graphical dependence was evident that can evaluate the mean value of $F_{r}$ and $F_{t}$. In the next step, the evaluation was drawn graph depending of the radial and tangential forces at a constant rotation $\omega$ rotor according to the precession of the rotor $\Omega$. For clarity, the boundary conditions are given including parameters setting numerical simulations for one variant, see Table 2.

Table 2. Boundary conditions, setting parameters

\begin{tabular}{|l|c|c|}
\hline Rotation & Value & Unit \\
\hline$n$ (rev) & 600 & $\mathrm{rev} / \mathrm{min}$ \\
\hline$n$ (rev) & 10 & $\mathrm{rev} / \mathrm{s}$ \\
\hline$\omega$ (angular velocity) & 62.8319 & $\mathrm{rad} / \mathrm{s}$ \\
\hline Precession & & \\
\hline$N$ (rev) & 600 & $\mathrm{rev} / \mathrm{min}$ \\
\hline$N$ (rev) & 10 & $\mathrm{rev} / \mathrm{s}$ \\
\hline$\Omega$ ( angular velocity) & 62.8319 & $\mathrm{rad} / \mathrm{s}$ \\
\hline$T$ (period, one orbit) & 0.1 & $\mathrm{~s}$ \\
\hline$T_{c}$ (total time) & 0.2 & $\mathrm{~s}$ \\
\hline$\Delta t$ (time step) & 0.000125 & $\mathrm{~s}$ \\
\hline
\end{tabular}

Evaluated value of the forces $F_{x}, F_{y}, F_{r}$ and $F_{t}$ for variant rotation $\omega=62.8319 \mathrm{rad} / \mathrm{s}$, and the precession $\Omega=$ $62.8319 \mathrm{rad} / \mathrm{s}$ rotor including mean values of forces $F_{r}$ and $F_{t}$ are listed in Table 3. In a similar manner were evaluated forces for different variants of combinations of 
speed and precession. The resulting distribution of radial force $F_{r}$ depending on the angle of rotation $\varphi$ for constant rotation $\omega=62.8319 \mathrm{rad} / \mathrm{s}$ and variable precession $(\Omega=$ $62.8319 \mathrm{rad} / \mathrm{s}, 125.6637 \mathrm{rad} / \mathrm{s}, 188.4956 \mathrm{rad} / \mathrm{s}, 251.3274$ $\mathrm{rad} / \mathrm{s}, 303.6873 \mathrm{rad} / \mathrm{s}, 314.1593 \mathrm{rad} / \mathrm{s}$ ) are shown in "Fig 9 ".

Table 3. Evaluation of the magnitude of forces $F_{x}, F_{y}, F_{r}$ and $F_{t}$ for variant $(\omega=62.8319 \mathrm{rad} / \mathrm{s}, \Omega=62.8319 \mathrm{rad} / \mathrm{s})$

\begin{tabular}{|c|c|c|c|c|c|}
\hline$[\varphi]={ }^{\circ}$ & {$[t]=\mathrm{s}$} & {$\left[F_{x}\right]=\mathbf{N}$} & {$\left[\boldsymbol{F}_{y}\right]=\mathbf{N}$} & {$\left[F_{r}\right]=\mathbf{N}$} & {$\left[F_{t}\right]=\mathbf{N}$} \\
\hline 45 & 0.0125 & 13.024 & -5.724 & -13.26 & -5.16 \\
\hline 90 & 0.025 & 12.992 & 5.166 & -12.99 & -5.17 \\
\hline 135 & 0.0375 & 5.671 & 12.969 & -13.18 & -5.16 \\
\hline 180 & 0.05 & -5.053 & 13.117 & -13.12 & -5.05 \\
\hline 225 & 0.0625 & -13.039 & 5.786 & -13.31 & -5.13 \\
\hline 270 & 0.075 & -12.986 & -5.174 & -12.99 & -5.17 \\
\hline 585 & 0.1625 & -13.029 & 5.759 & -13.29 & -5.14 \\
\hline 630 & 0.175 & -12.974 & -5.142 & -12.97 & -5.14 \\
\hline 675 & 0.1875 & -5.673 & -12.985 & -13.19 & -5.17 \\
\hline 720 & 0.2 & 5.019 & -13.014 & -13.01 & -5.02 \\
\hline \multicolumn{4}{|c|}{ Mean value } & -13.11 & -5.12 \\
\hline
\end{tabular}

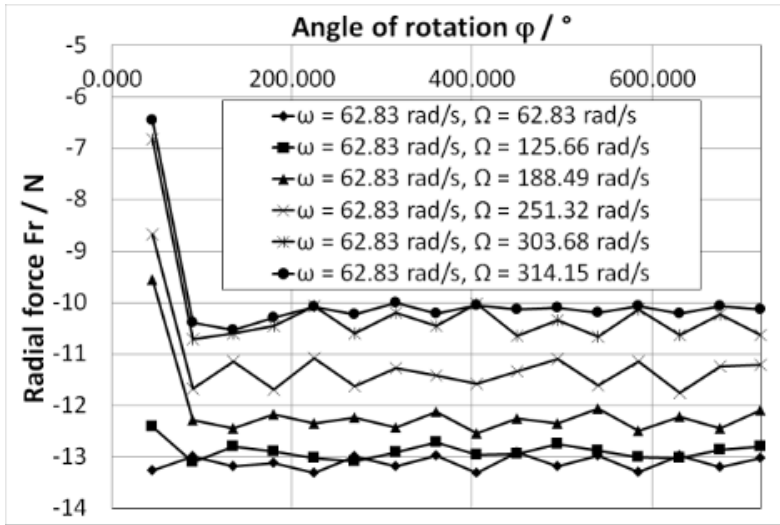

Fig. 9. Distribution of radial force $F_{r}$ depending on the angle of rotation $\varphi$ for constant rotation $\omega$ and variable precession $\Omega$

In the same way the courses were evaluated tangential forces $F_{t}$, see figure 10. A similar procedure was then applied for other values of rotation $\omega$.

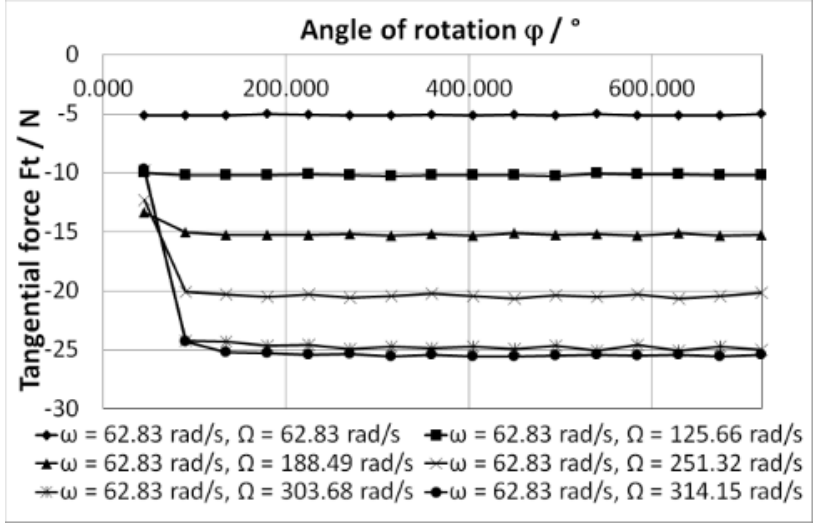

Fig. 10. Distribution of tangential force $F_{t}$ depending on the angle of rotation $\varphi$ for constant rotation $\omega$ and variable precession $\Omega$

\subsection{Evaluation of distribution $F_{r}$ a $F_{t}$}

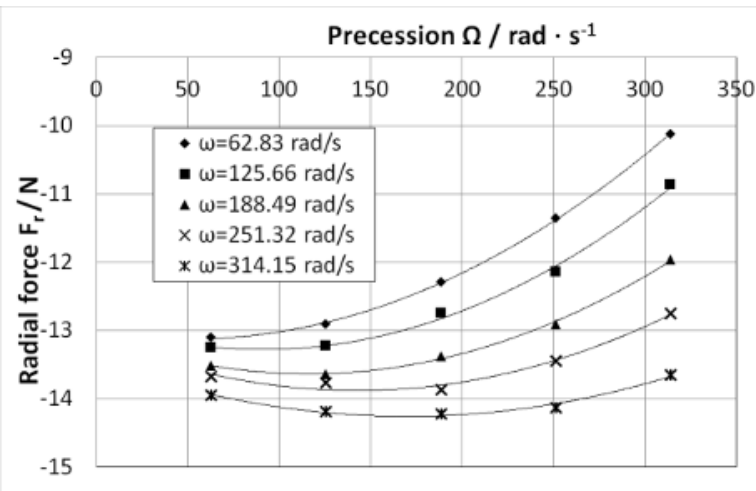

Fig. 11. Distribution of radial force $F_{r}$ depending on the precession $\Omega$ for constant rotation $\omega$

From the evaluated functional dependence (figure 11) were set polynomial regression dependencies:

$$
F_{r(\omega)}=A+B \cdot \Omega+C \cdot \Omega^{2}
$$

Thus

$$
\begin{aligned}
& F_{r(\omega=62.8319 \mathrm{rad} / \mathrm{s})}=4.350 \cdot 10^{-5} \Omega^{2}-4.436 \cdot 10^{-3} \Omega-13.0115 \\
& F_{r(\omega=125.663 \mathrm{rad} / \mathrm{s})}=4.769 \cdot 10^{-5} \Omega^{2}-8.687 \cdot 10^{-3} \Omega-12.8838 \\
& F_{r(\omega=188.495 \mathrm{rad} / \mathrm{s})}=4.227 \cdot 10^{-5} \Omega^{2}-9.831 \cdot 10^{-3} \Omega-13.0631 \\
& F_{r(\omega=251.3274 \mathrm{rad} / \mathrm{s})}=3.794 \cdot 10^{-5} \Omega^{2}-1.084 \cdot 10^{-2} \Omega-13.1059 \\
& F_{r(\omega=314.1593 \mathrm{rad} / \mathrm{s})}=2.815 \cdot 10^{-5} \Omega^{2}-9.560 \cdot 10^{-3} \Omega-13.4463
\end{aligned}
$$

In the Table 4 are summarized the values of the coefficients of polynomial relations $A, B$ and $C$, which can be drawn in dependence on the rotation $\omega$ (figure 12, figure 13, figure 14).

Table 4. Evaluation of the coefficients $(A, B, C)$ for the polynomial depending

\begin{tabular}{|c|c|c|c|}
\cline { 2 - 4 } \multicolumn{1}{c|}{} & $\mathbf{A}$ & $\mathbf{B}$ & $\mathbf{C}$ \\
\hline$[\omega]=62.8319 \mathrm{rad} / \mathrm{s}$ & -13.0115 & -0.00443615 & $4.35 \mathrm{E}-05$ \\
{$[\omega]=125.6637 \mathrm{rad} / \mathrm{s}$} & -12.8838 & -0.00868725 & $4.77 \mathrm{E}-05$ \\
{$[\omega]=188.4956 \mathrm{rad} / \mathrm{s}$} & -13.0631 & -0.0098311 & $4.23 \mathrm{E}-05$ \\
{$[\omega]=251.3274 \mathrm{rad} / \mathrm{s}$} & -13.1059 & -0.0108397 & $3.79 \mathrm{E}-05$ \\
{$[\omega]=314.1592 \mathrm{rad} / \mathrm{s}$} & -13.4463 & -0.0095603 & $2.81 \mathrm{E}-05$ \\
\hline
\end{tabular}

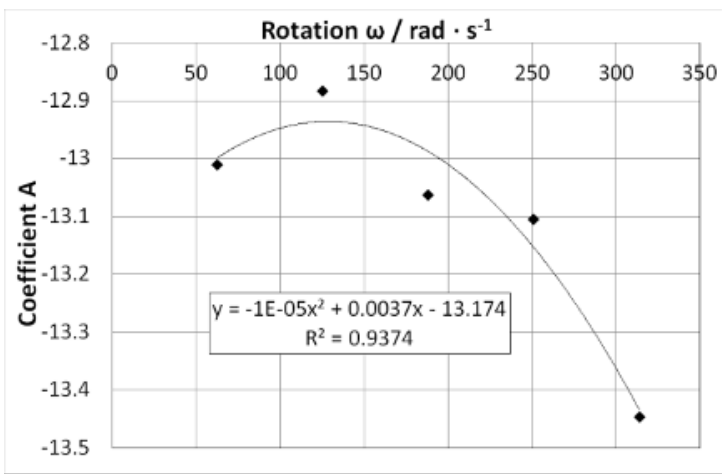

Fig. 12 Course of the coefficient $A$ in depending on the rotation $[\omega]=\mathrm{rad} / \mathrm{s}$ 


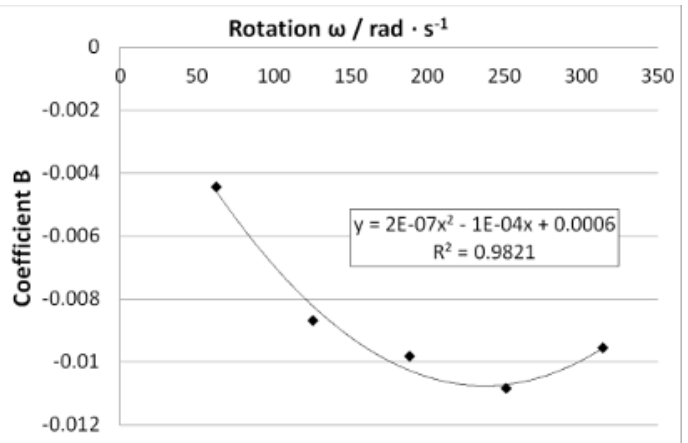

Fig. 13 Course of the coefficient $B$ in depending on the rotation $[\omega]=\mathrm{rad} / \mathrm{s}$

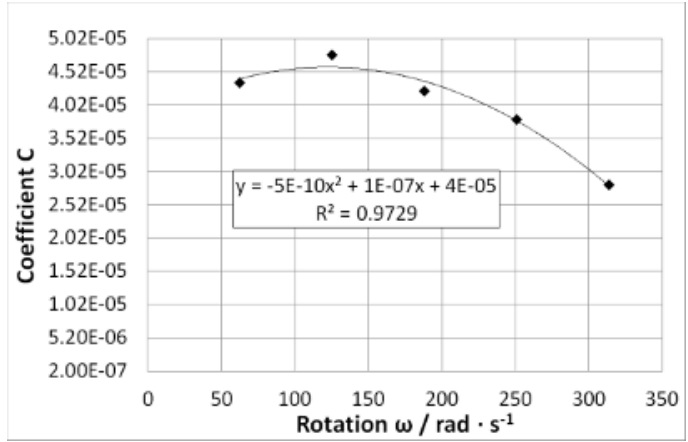

Fig. 14 Course of the coefficient $\mathrm{C}$ in depending on the rotation $[\omega]=\mathrm{rad} / \mathrm{s}$

\subsection{Graphical evaluation of current values}

Indisputable advantage of CFD modeling is the possibility to view detailed current calculated quantities (pressure, speed, ...). The following sections are evaluated static pressure distribution in different sections and areas of the computational domain. In the 3D model was created two evaluation levels, see figure 15 . It is a longitudinal plane and a transverse plane, which are led computational domain.

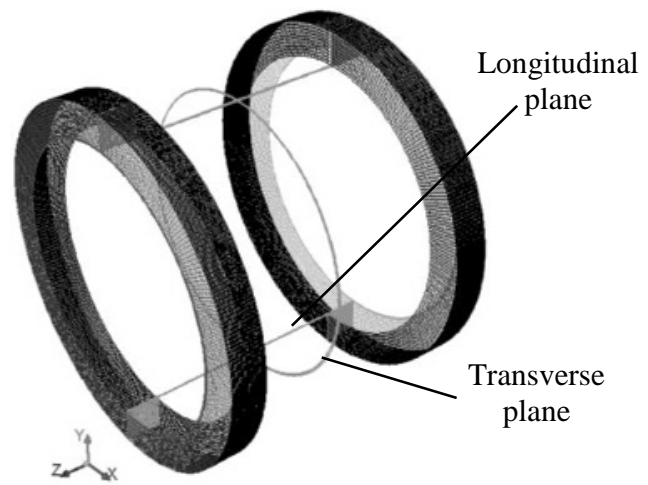

Fig. 15 Evaluation plane

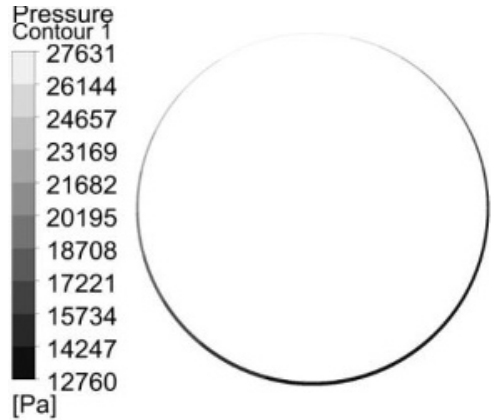

Fig. 16 Filled contours of static pressure in the transverse plane
Evaluation of static pressure is made for rotation variant $\omega=125.6637 \mathrm{rad} / \mathrm{s}$, and the precession $\Omega=62.8319 \mathrm{rad} / \mathrm{s}$ after passing two precession speed rotor. Of course static pressure figure 17 is evident inlet pressure $10^{5} \mathrm{~Pa}$ and pressure drop in the course of a narrow gap to zero value. The figure 18 shows the distribution of the static pressure on the wall of the rotor. Again, it is evident from the picture input pressure and a gradual decrease in pressure.

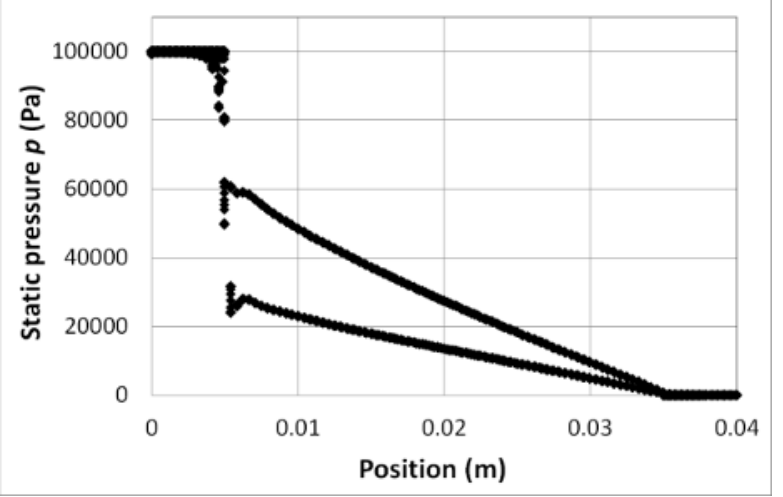

Fig. 17 Course of the static pressure in a longitudinal section

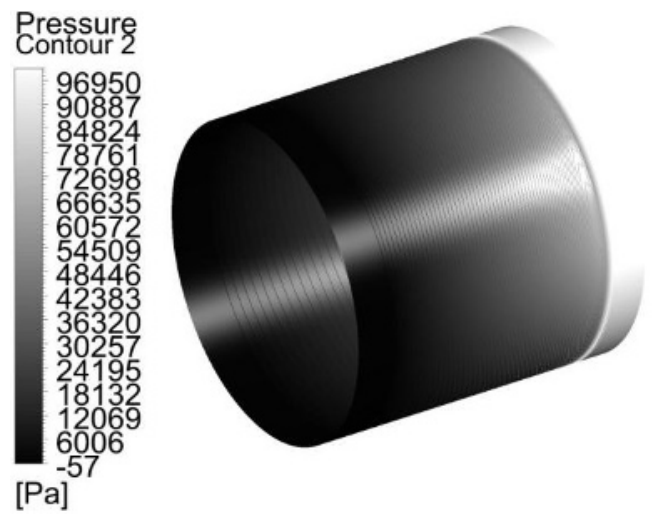

Fig. 18 The distribution of static pressure on the wall of the rotor

\section{Conclusions}

The article defines the problem of mathematical modeling of flow in the narrow gap between the two cylinders, when the inner cylinder is eccentrically placed and held simultaneously rotating and precession motion in a circle. From the result of numerical simulation using ANSYS Fluent software are determined the radial and tangential forces and other dependencies. As defined above approach can be applied for example in the case of a hydrostatic bearing lubrication, flow modeling sealing gaps pumps or turbines, and many other technical applications.

\section{References}

1. H. Black, J. Mech. Eng. Scien. 11, 8 (1969)

2. Y. Kanemori, T. Iwatsubo, J. of Trib. 114, 6 (1992)

3. T. Staubli, B. Matthias, Int. Symp. On St. Noc. Of Rot. Mach. (ISCORMA), (2001)

4. ANSYS Fluent 13 - User's Guide. Fluent Inc, 2010

5. M. Kozubková, J. Rautová, F. Pochylý. Acta Hyd. Pneu. 5, 8 (2008)

6. R. B. Bird, W. E. Stewart, E. N. Lightfoot. Transport Phenomena. John Wiley \& Sons. 895 (2001) 